\title{
Magnetic Resonance Imaging of Neuronal Function in the Spinal Cord: Spinal fMRI
}

\author{
Patrick W. Stroman, PhD
}

\begin{abstract}
A review of the current literature on magnetic resonance imaging of neuronal function in the spinal cord (spinal fMRI) is presented. The unique challenges of spinal fMRI are identified as being the small cross-sectional dimensions of the spinal cord, magnetic field inhomogeneity caused by the bone and cartilage in the spine, and motion of cerebrospinal fluid, blood, adjacent tissues and organs and of the spinal cord itself. Techniques have been developed to overcome or compensate for these challenges and the result is a fMRI method which is distinct from that used for mapping function in the brain. Evidence that the current spinal fMRI method provides accurate and sensitive maps of neuronal function is also discussed. Studies presented in the literature have demonstrated areas of neuronal activity corresponding with spinal cord neuroanatomy as a result of thermal and electrical stimuli and motor tasks with the hands, arms and legs. Signal intensity changes detected in active areas have also been demonstrated to depend on the intensity of the stimuli with both thermal stimulation and a motor task, providing evidence of the correspondence between spinal fMRI results and neuronal activity in the spinal cord.
\end{abstract}

Keywords: Animals; fMRI; Humans; Magnetic resonance imaging; Motor; functional MRI; Pain; Reflex; Sensory; Spinal cord; Spinal cord injuries

F spinal cord has been developed over the past 7 years and now appears to be adequate for practical use as a research tool and for clinical trials assessing spinal cord function. The magnetic resonance imaging (MRI) method for demonstrating areas of neuronal activity in the brain has received a great deal of attention and has developed at a rapid pace since it was first demonstrated in 1990. ${ }^{1-3}$ The method has been proven to be a powerful tool for mapping neuronal function with several hundred scientific papers published each year related to fMRI of the brain. The application of fMRI to the spinal cord (spinal fMRI) seems a logical extension of its use in the brain, but in comparison has received relatively little attention. To date there have been approximately 17 scientific papers published on spinal fMRI in humans and animals. The relatively low number of publications appears to be, at least in part, a consequence of the considerable challenge of acquiring MRIs of the spinal cord, in addition to the usual challenges of obtaining high quality fMRI data. Nonetheless, the work that has been published and presented at scientific meetings has

Reprint Requests: Patrick W. Stroman, PhD, Department of Diagnostic Radiology, c/o Center for Neuroscience Studies, 23I Botterell Hall, Queen's University, Kingston, Ontario, Canada K7L 2V7; Tel: 6I3-533-3245;

Fax: 613-533-6840; Email: stromanp@post.queensu.ca. shown consistent findings across several groups that are now working on spinal fMRI and demonstrates that the challenges of applying fMRI to the spinal cord can be overcome. Most of the work that has been published with human subjects demonstrates that spinal fMRI can be carried out using current clinical MRI systems without any custom hardware or software.

\section{Rationale for Developing Spinal fMRI}

The need for an fMRI method adapted for demonstrating function in the spinal cord arises from the fact that the cord is contained within the vertebral column and is therefore relatively inaccessible. Without opening the spinal canal or risking injury to the cord by inserting electrodes or needles, the only means of assessing the function of the cord relies on the patient's (or research animal's) ability to feel a stimulus or to have an overt reflex response. This requires the assumption that the sensory receptors, peripheral nerves and relevant areas of the brain are all functioning normally. Even if this assumption is correct, very little information can be obtained about the condition of the spinal cord caudal to an injury site. This effectively hides very important physical and 
physiological information that may be needed for proper assessment of a patient's condition or the effectiveness of treatment.

\section{Challenges}

Most challenges of applying fMRI methods to the spinal cord also arise from the fact that the cord is contained within the vertebral column. Differences in the magnetic susceptibilities between the bone, cartilage and tissues result in subtly different magnetic fields within these materials and field gradients at their boundaries. These gradients can cause distortion and loss of signal in MRIs and present a challenge for obtaining good image quality in the spinal cord. A similar effect arises from the lungs, which are near to the cord over most of its length, with the added complication that the field distortions they cause fluctuate rhythmically with breathing. The cerebrospinal fluid (CSF) that flows around the cord within the spinal canal presents another challenge because of the motion artifacts produced, as well as from the motion this imparts to the spinal cord. Finally, the $45 \mathrm{~cm}$ average length of the cord and its relatively small cross-section (approximately $16 \mathrm{~mm} \times 10 \mathrm{~mm}$ in the cervical enlargement) present another problem for MRI because a trade-off is required between image resolution and the volume of tissue that can be imaged in a reasonable amount of time (preferably $\leq 10 \mathrm{sec}$ ).

\section{Problems of Field Homogeneity and Image Quality}

The first published example of fMRI in the spinal cord was by Yoshizawa et al. ${ }^{4}$ in 1996 after blood-oxygen-level-dependent (BOLD) fMRI of the brain had already been established. This work and the earliest attempts by other groups applied the established brain fMRI methods of the time to the spinal cord.
The consistent features of the studies by Yoshizawa et al., ${ }^{4}$ Stroman et al., ${ }^{5}$ Madi et al. ${ }^{6}$ and Backes et al. ${ }^{7}$ were that they were carried out with healthy volunteers and employed a hand motor task with imaging of the cervical spinal cord. All used gradient-echo methods with echo times of 40-50 msec at 1.5 $\mathrm{T}$ and $31 \mathrm{msec}$ at $3 \mathrm{~T}$, as is typical with brain $\mathrm{fMRI}$. The areas of activity in the spinal cord demonstrated by these studies corresponded to the expected areas of neuronal activity with the stimuli applied. In each of these manuscripts the authors concluded that spinal fMRI is a feasible method for assessing neuronal activity in the cord. However, the results obtained also demonstrated variability in the areas of activity, and that it is difficult to obtain high quality fMRI data in the spinal cord with gradient-echo methods and sensitivity to the BOLD effect.

The nature of the signal changes in the spinal cord were investigated by comparing $\mathrm{T}_{2}$-weighted and $\mathrm{T}_{2}{ }^{*}$-weighted spinal fMRI data obtained with spin-echo imaging and gradient-echo imaging (echo-planar imaging in both cases), respectively (figure 1). ${ }^{8}$ The fMRI studies carried out in the brain have demonstrated that the ratio of signal changes with gradient-echo data to those with spin-echo data should be at least 3.5:1 when acquired at the same echo time. ${ }^{9}$ This comparison in the spinal cord demonstrated a ratio of signal changes that is slightly $<1: 1$ and is inconsistent with the BOLD theory. Both the spin-echo and gradient-echo data demonstrated signal changes correlated with the stimulation paradigm and both demonstrated signal changes that increased with the echo time. ${ }^{8}$ These points are consistent with the data demonstrating a BOLD effect. However, the signal change extrapolated to an echo time of zero (no sensitivity to relaxation times or changes in this property) did

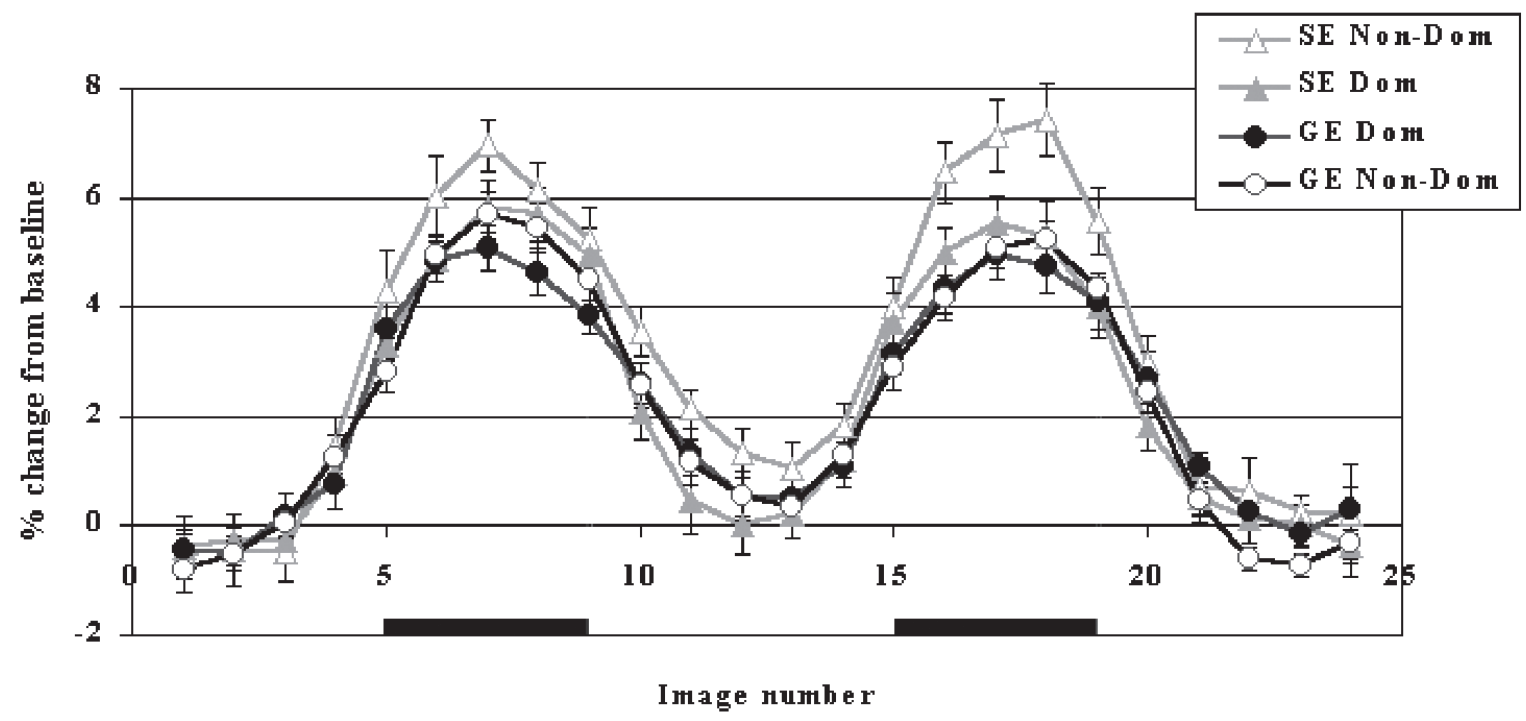

Figure 1. Signal intensity time courses observed with a one-hand motor task (ball squeezing) with gradient-echo (GE) and spin-echo (SE) image acquisition at 1.5 tesla. Black lines and symbols indicate gradient-echo data, whereas gray lines and symbols indicate spin-echo data with the dominant and non-dominant hands. The exercise periods of the stimulation paradigm are indicated with the solid black bars at the bottom of the plot. Error bars indicate the standard error of the mean (SEM). 
not approach zero with the spin-echo data, but was consistently around $2.5 \%$, and this is not consistent with the BOLD effect. A detailed study which followed demonstrated that the relationship between the fractional signal change $(\triangle \mathrm{S} / \mathrm{S})$ and echo times for spin-echo data was non-linear, as shown in figure 2.10 At an echo time of only $11 \mathrm{msec}$, the average signal intensity change was $3.3 \%$ with spin-echo imaging. This is proton-density weighted data, as there is very little sensitivity to transverse relaxation $\left(\mathrm{T}_{2}\right)$, and the signal changes cannot be attributed to the BOLD effect. While this raised significant questions regarding the mechanism of signal change, an immediate conclusion that was demonstrated by this observation is that spinal fMRI can be carried out with proton-density weighted spin-echo imaging. This imaging technique provides the highest image quality in the spinal cord because it has the least sensitivity to spatial variations in the static magnetic field arising from magnetic susceptibility differences.

The fundamental problem of poor magnetic field homogeneity in the spinal cord was, therefore, demonstrated to be overcome with the use of spin-echo imaging methods with short echo times. Although this method does not provide high sensitivity to the BOLD effect, it nonetheless demonstrates areas of signal change corresponding to areas of neuronal activity in the spinal cord. The current evidence supporting this conclusion is discussed below.

\section{Contrast Mechanism for Spinal fMRI}

The mechanism of signal change in the spinal cord has been demonstrated to have a contribution from the BOLD effect, as well as from a proton-density change at sites of neuronal activity. ${ }^{10}$ However, the contribution of a proton-density change had not been previously demonstrated in fMRI data, and its relationship to neuronal activity was unknown. The detailed study of the dependence of the fractional signal change $(\triangle \mathrm{S} / \mathrm{S})$ with the echo time discussed above, demonstrated that the proton-density change contribution likely occurs in a water component that is separate from that in which the BOLD effect occurs. ${ }^{10}$ As the BOLD effect arises from the blood, ${ }^{1-3}$ this finding is consistent with the proton-density change occurring outside the vascular spaces and so it was termed "SEEP" for "Signal Enhancement by Extravascular water Protons." A two-component model was developed that, when fit to the data, demonstrated one component with a $\mathrm{T}_{2}$ value changing from $172 \pm 9 \mathrm{msec}$ during rest conditions to $200 \pm 13 \mathrm{msec}$ upon stimulation. ${ }^{10}$ These values are consistent with the BOLD effect showing the $\mathrm{T}_{2}$ of blood in the capillaries having an oxygenation of approximately $80 \%$ at rest and increasing to $85 \%$ during stimulation. ${ }^{11}$ The second component exhibited a constant $\mathrm{T}_{2}$ of $71 \pm 21 \mathrm{msec}$ (consistent with gray matter) that did not change upon stimulation, and a proton-density which increased by $5.6 \% \pm 0.2 \%$ upon stimulation. These observations are again consistent with the proton-density change occurring within an extravascular water component.

Studies were carried out to further investigate this effect employing fMRI of the brain to permit comparisons with the broader base of brain fMRI results in the literature. Under similar experimental conditions (i.e., spin-echo data; proton-density weighted: echo times $=21$ to $23 \mathrm{msec}$; sensory stimulation of the hand) signal intensity changes in the sensory areas of the brain in healthy volunteers were observed to be $1.9 \% \pm 0.3 \%$ at 3 tesla, ${ }^{12} 1.9 \% \pm 0.5 \%$ at 1.5 tesla, ${ }^{13}$ and $1.7 \% \pm 0.1 \%$ and $2.0 \% \pm 0.2 \%$ at 0.2 tesla. ${ }^{14}$ The BOLD

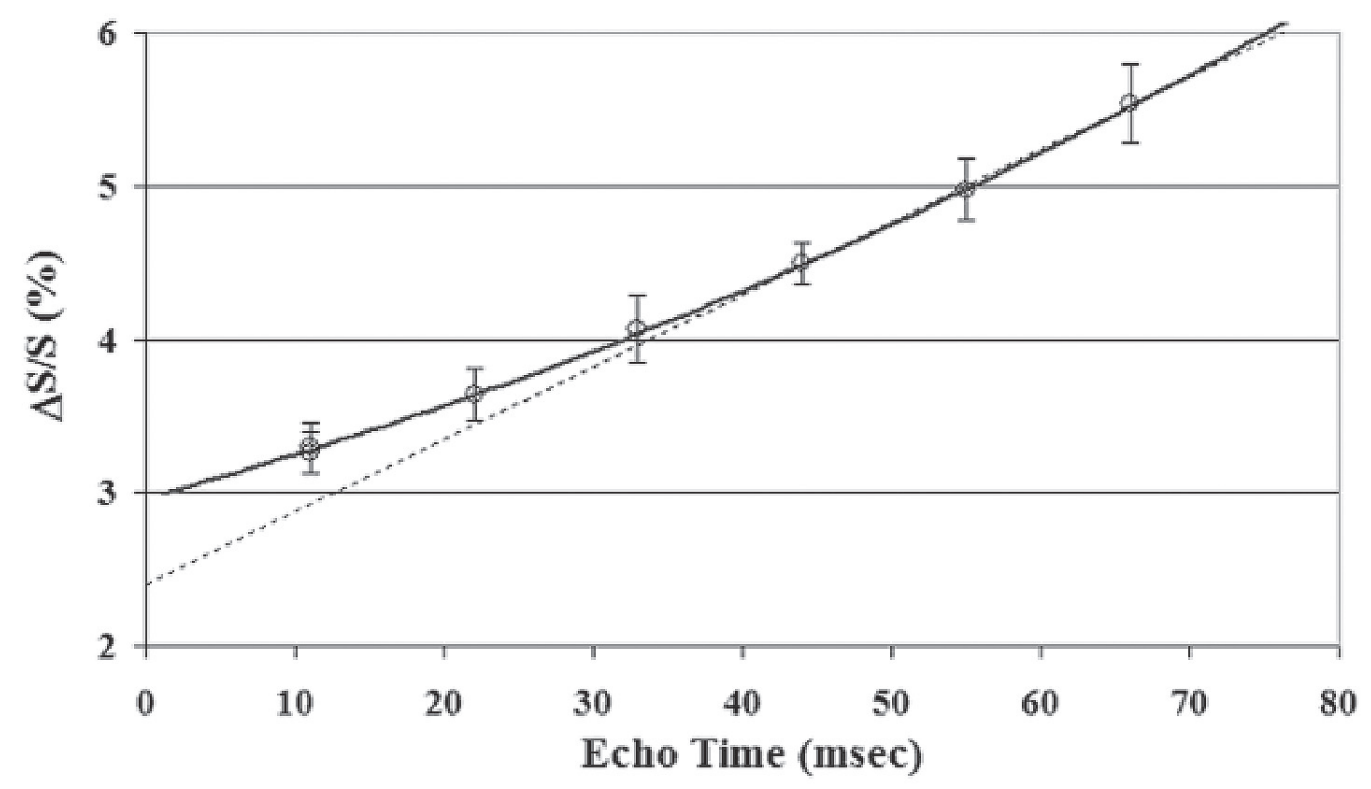

Figure 2. Fractional signal changes $(\Delta S / S)$ observed with spin-echo fMRI data in the cervical spinal cord as a function of echo time at 1.5 tesla. Data are average values from 15 healthy volunteers. Average values for the $1^{\text {st }}$ and $2^{\text {nd }}$ measures obtained with echo time $=11 \mathrm{msec}$ are plotted separately. The solid line demonstrates the result of fitting with a non-linear model, whereas the dashed line demonstrates a linear fit to data with echo time $>33$ msec only. Error bars indicate the standard error of the mean. 
effect has been shown to depend on the magnetic field strength ${ }^{15}$ and is expected to be negligible in the spin-echo data acquired at $0.2 \mathrm{~T}$. These studies confirm that there is a non-BOLD contrast mechanism that does not arise from relaxation time changes and is consistent only with a proton-density change.

Comparisons were also made between BOLD and SEEP fMRI results obtained at $1.5 \mathrm{~T}$ and $3 \mathrm{~T}$. These studies consistently demonstrated that areas of SEEP activity were immediately adjacent to areas of BOLD activity with very little overlap. ${ }^{12,13}$ While SEEP and BOLD contrast mechanisms are observed simultaneously, their primary sites of origin do not appear to be at the same anatomical locations.

\section{Proposed Physiological Origins of SEEP}

At sites of neuronal activity there are well-known physiological processes occurring in addition to the BOLD effect. It is well established that local hemodynamic changes result in increased blood flow and increased perfusion pressure at sites of neuronal activity. ${ }^{16}$ Such increases may also increase the normal rate of production of extracellular fluid arising from water being exuded from the blood vessels. Positron emission tomography (PET) studies using a radiolabeled water tracer have demonstrated that there is increased unidirectional clearance of water from the blood into the tissues at sites of neuronal activity in the brain. ${ }^{17,18}$

Another factor influencing the local fluid balance is cellular swelling at sites of activity. Astrocytes make contact with both blood vessels and neurons and play a role in providing metabolites to neurons and in maintaining the extracellular concentration of glutamate. ${ }^{19-21}$ As a result, these cells have been shown to play an important role in effective neuronal signaling. ${ }^{22,23}$ When glutamate is released from vesicles in the axon terminal and travels across the synaptic cleft to trigger the depolarization of the adjoining neuron, the remaining glutamate is rapidly absorbed by astrocytes by means of high-affinity sodium-dependent transporters. ${ }^{22}$ When glutamate is actively taken up by astrocytes, each molecule is accompanied by 3 $\mathrm{Na}^{+}$and $1 \mathrm{H}^{+}$transported into the cell and $1 \mathrm{~K}^{+}$transported out. This ionic exchange is also accompanied by water co-transport into the cells. ${ }^{22}$ In addition, astrocytes have been shown to be depolarized during neuronal activity as a result of potassium uptake in proportion to the number of active neurons in the vicinity and the frequency at which they fire. ${ }^{20}$ The flux of potassium across the astrocyte plasma membrane is coupled with water transport via specific channels known as aquaporins (specifically Aqp4). ${ }^{24}$ These channels exist throughout the brain but are concentrated in the membranes of astrocyte end-feet, which face blood vessels. There is strong evidence that these end-feet promote the exchange of water between the brain and blood or CSF. As a result of these effects, the extracellular/intracellular volume ratio has been shown to change significantly in isolated tissue samples of rat spinal cord tissue during and following neuronal stimulation. ${ }^{25,26}$
With glial cells being 5 to 10 times more numerous than neurons and occupying roughly half the volume of the central nervous system, they contribute a significant portion of the MRI signal. Moreover, astrocytes are connected by gap junctions, allowing passage of small molecules from cell to cell and, thus, function as a continuous, large fluid volume. ${ }^{27}$ Given the role astrocytes play in maintaining the extracellular environment for effective neuronal signaling and in providing metabolites, these cells may play a very significant role in fMRI as well. Evidence for this is demonstrated in the neuroscience literature where it is generally well accepted that brief neuronal discharge leads to sustained swelling of adjacent glial cells. For example, changes in neuronal and glial cell volumes related to neuronal activity cause changes in light transmittance and are used for imaging activity in spinal cord 25,28 and brain slices. ${ }^{29,30}$ Rat brain slices submerged in flowing artificial CSF solution demonstrated increased light transmittance as a result of cell swelling, specifically at sites where electrically stimulated fibers terminated on dendrites in the hippocampus. ${ }^{30}$ Although no proof has yet been obtained that the production of extracellular fluid and glial cell swelling are related to the observed SEEP contrast mechanism, the data obtained to date support the hypothesis that these processes play a role.

\section{Problems Associated with Small Physical Dimensions of the Cord and Partial Volume Effects}

The small physical dimensions of the spinal cord and the distribution of white and gray matter and the surrounding CSF create a requirement for high resolution data (preferably voxel dimensions $<2 \mathrm{~mm} \mathrm{~A} / \mathrm{P}$ by $2 \mathrm{~mm} \mathrm{R} / \mathrm{L}$ ) in order to avoid mixing of gray matter, white matter and/or CSF in each voxel. However, the data must also have a sufficiently high signal-to-noise ratio. The signal from each voxel depends on the volume of tissue/fluid it contains, and larger voxel dimensions provide a higher signal-to-noise ratio. An optimal balance of slice orientation, image resolution and slice thickness must therefore be determined to achieve both adequate resolution and signal-to-noise ratio.

Published studies that have reported success at detecting areas of neuronal activity in human subjects with either gradient-echo or spin-echo methods have generally employed transverse slices with an in-plane resolution of $2 \mathrm{~mm}$ or less. A greater degree of sensitivity is evident in the reported studies with finer resolution, the majority of which have been carried out with a roughly $1 \mathrm{~mm}$ in-plane resolution or less. This resolution is adequate for distinguishing gray and white matter regions and is necessary for clear identification of gray matter regions involved in a task or stimulus. However, many studies with transverse slices have employed a slice thickness of up to $10 \mathrm{~mm}$ with reportedly good sensitivity in the results obtained.

More consistently reliable results have been demonstrated to date with transverse slices than with sagittal ones, but the advantage of greater volume coverage with sagittal or coronal slices is clear. The use of coronal slices has been successfully demonstrated in animal spinal fMRI studies using spin-echo 
imaging methods. ${ }^{31}$ However, the curvature of the spinal cord is expected to be problematic in this orientation in human studies; it is likely that a coronal slice will not demonstrate a consistent anterior-posterior plane of the spinal cord. Because there is generally very little right-left curvature of the spinal cord, sagittal slices are expected to demonstrate a consistent portion of the cord, but will nonetheless suffer from partial volume effects, unless the slice thickness is as thin as possible. Studies employing sagittal slices have been reported in humans using slices ranging from $4 \mathrm{~mm}$ to $8 \mathrm{~mm}$ thick. ${ }^{5-7}$ However, the results have been variable with less consistent correspondence to the spinal cord neuroanatomy and a wide range of signal intensity changes reported at $0.5 \%-7.5 \%, 64.3 \%-4.5 \%, 5$ and $8 \%-12 \%$. $^{7}$ Given that the human spinal cord is roughly 16 $\mathrm{mm}$ across at its widest point, the sagittal data that have been presented clearly suffer from significant partial volume effects and reduced sensitivity to the true neuronal activity-related signal changes.

In order to obtain reliable data in a sagittal orientation, it has been proposed that one can acquire contiguous sagittal slices with the thickness set to the minimum that can be achieved by the MRI hardware, regardless of the signal-to-noise ratio. ${ }^{32}$ By reformatting these data into a three-dimensional (3D) volume, one can smooth across uniform tissue volumes by applying the smoothing only in the rostral-caudal direction along the spinal cord. This serves to increase the signal-to-noise ratio without incurring significant partial volume effects. In order to test this method, data were acquired in repeated studies of 7 healthy volunteers. Cold thermal stimulation of the palm of the hand was employed to elicit activity in the cervical spinal cord without inducing task-related motion and to allow comparisons with previous studies. Prior to analysis, a mid-line sagittal slice was selected from each data set and a reference line was drawn manually along the anterior edge of the cord. The data were then reformatted into a 3D volume, interpolated into small isotropic voxels, and the volume was re-sliced perfectly transverse to the cord at each point along the reference line in the rostral-caudal direction. The reference line was also used to align the anterior edge of the cord in each resulting transverse slice. The data were smoothed only across adjacent transverse slices (i.e., smoothed in the rostral-caudal direction) and a correlation analysis was applied as in previous studies. The resulting data demonstrated details of the distribution of activity at the sub-segmental level; small ( $\sim 2 \mathrm{~mm}$ to $3 \mathrm{~mm}$ ) localized areas of activity and a pattern of dorsal and ventral activity consistent with the stimulus and with previous studies were observed. The magnitude of signal intensity changes was larger than observed in previous studies at $10 \% \pm 1 \%$ (mean \pm S.D.) suggesting that partial-volume effects had been further reduced by the increased resolution in the rostral-caudal direction. The data were also reformatted to match the volumes spanned by $7.5 \mathrm{~mm}$ thick transverse slices and reanalyzed for comparison with previous studies. This resulted in active voxels being identified with signal changes of $6.3 \% \pm 0.5 \%$ in comparison with $6.4 \% \pm 1.3 \%$ observed in studies employing transverse slices and similar stimulation conditions. ${ }^{10,33,34}$ The added benefit of this sagittal-slice method is that it provides a full three-dimensional map of activity across a $12 \mathrm{~cm}$ length of the spinal cord enabling results to be displayed in any desired slice orientation, as well as demonstrating surrounding anatomical landmarks such as spinal nerves, vertebrae and spinous processes. The resulting effective resolution obtained with this method was $2.8 \mathrm{~mm}$ x $0.9 \mathrm{~mm} \times 1.8 \mathrm{~mm}(\mathrm{R} / \mathrm{L} \times \mathrm{A} / \mathrm{P} \times \mathrm{S} / \mathrm{I})$ and the voxel volume and signal to noise ratio were similar to that obtained with transverse slices and voxel dimensions of $0.94 \mathrm{~mm} \times 0.94 \mathrm{~mm}$ $\mathrm{x} 7.5 \mathrm{~mm}$.

\section{Problems Arising from Motion}

The detection of MRI signal intensity changes arising in relation to a change in neuronal activity requires that each image voxel in a time-series data set represents the same tissue volume at each time point. Slight movement can be tolerated, particularly if the volume spanned by neighboring voxels also contains active tissue. In the spinal cord, however, the spatial extent of gray matter regions is relatively small, therefore very little motion can be tolerated. Confounding the problem is the pulsatile flow of CSF which causes the spinal cord to move slightly with each heartbeat. Another problem arises when nearby tissues move and create image artifacts that contribute, in error, to the signal within a voxel spanning a volume of the spinal cord. This effect can arise from the motion of CSF, lungs, heart or other organs or tissues.

It has been shown that some motion of the spinal cord may arise from blood flow in the radicular arteries, ${ }^{35}$ but the bulk of the motion is expected to be caused by CSF flow. ${ }^{36,37}$ The magnitude of displacement depends on the rostral-caudal position with the cord and on its curvature, as can be observed in serial images of the spinal cord with sagittal slices.

Various means of compensating for these sources of motion have been applied in virtually every spinal fMRI study reported to date, but the relative importance of each is only now becoming apparent as more studies are reported. Reduction of image artifacts arising from moving tissues and fluids has been successfully achieved with the use of flow-compensation gradients applied in the rostral-caudal direction and spatial saturation pulses to eliminate all signal arising from anterior to the spine. $10,33,38,39$ These serve to eliminate sources of signal from areas outside of the spinal cord that could contribute to voxels within the spinal cord as a result of spatial encoding errors created by motion. Respiratory-gated acquisition and breath-hold during acquisition have also been employed to reduce or eliminate the effects of lung motion. 5,34 However, in the studies carried out in our lab with proton-density weighted spin-echo imaging there was no apparent benefit or improvement in data quality obtained from respiratory gating or breath-holding. In one of the earliest spinal fMRI studies reported, Backes et al. ${ }^{7}$ applied cardiac-gated acquisition. However, this had not been investigated again until a comparison was made by Brooks et 

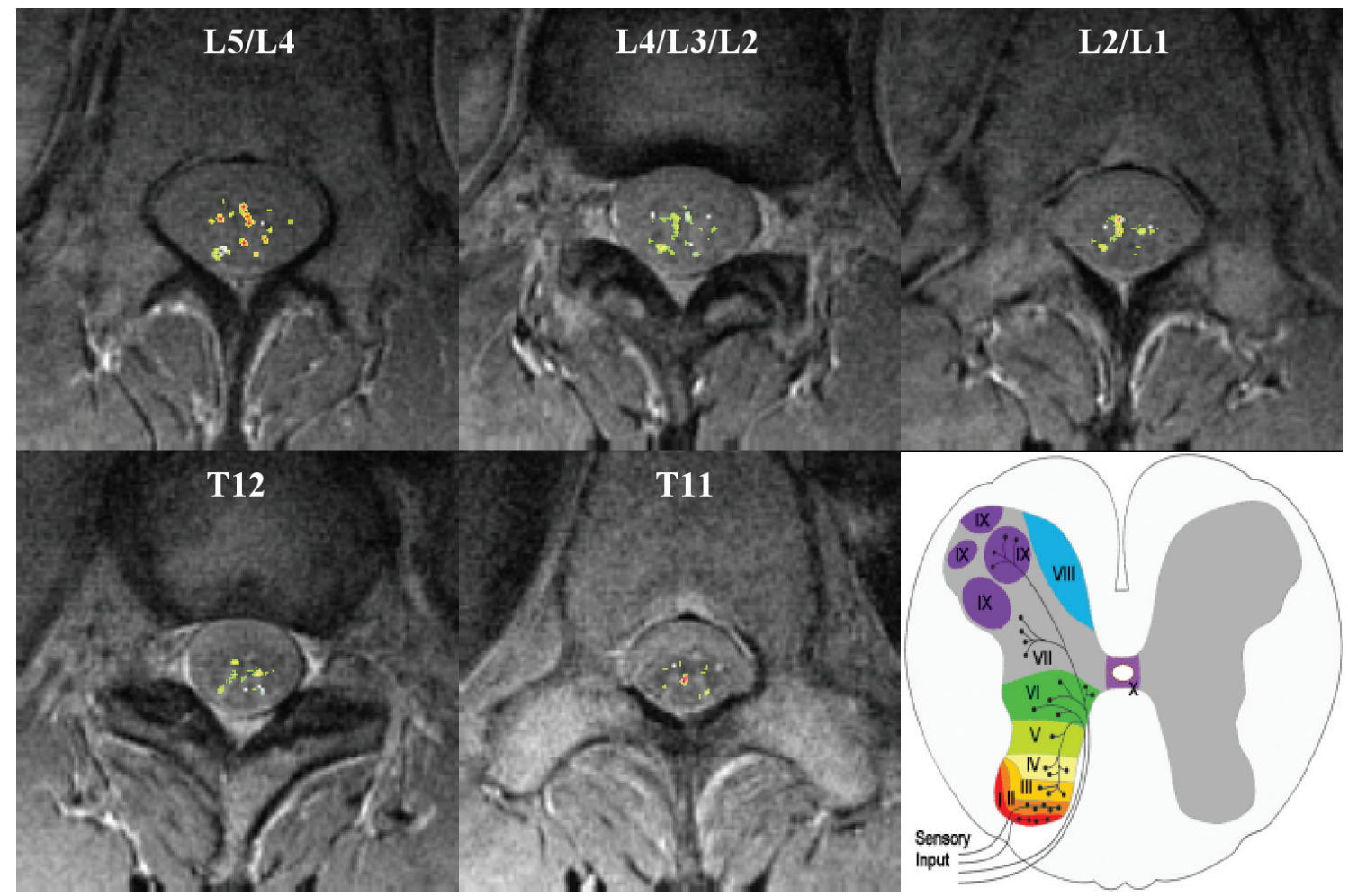

Figure 3. Activity in the lumbar spinal cord in response to a $10^{\circ} \mathrm{C}$ thermal stimulus applied to the L4 dermatome on the right leg (medial to anteromedial skin roughly $10 \mathrm{~cm}$ below the knee). The images are overlaid with the combined activity from 13 subjects demonstrating the consistent areas of activity. Red indicates the greatest consistency (greatest overlap) across subjects with the degree indicated by colors decreasing in spectral order. The schematic inlay (bottom right) shows the expected areas of activity in the spinal cord with a sensory or noxious stimulus. The approximate spinal cord segmental levels that are represented are indicated at the top of each frame. Images are in radiological orientation with the right side of the body to the left and the dorsal aspect of the spine toward the bottom of the images.

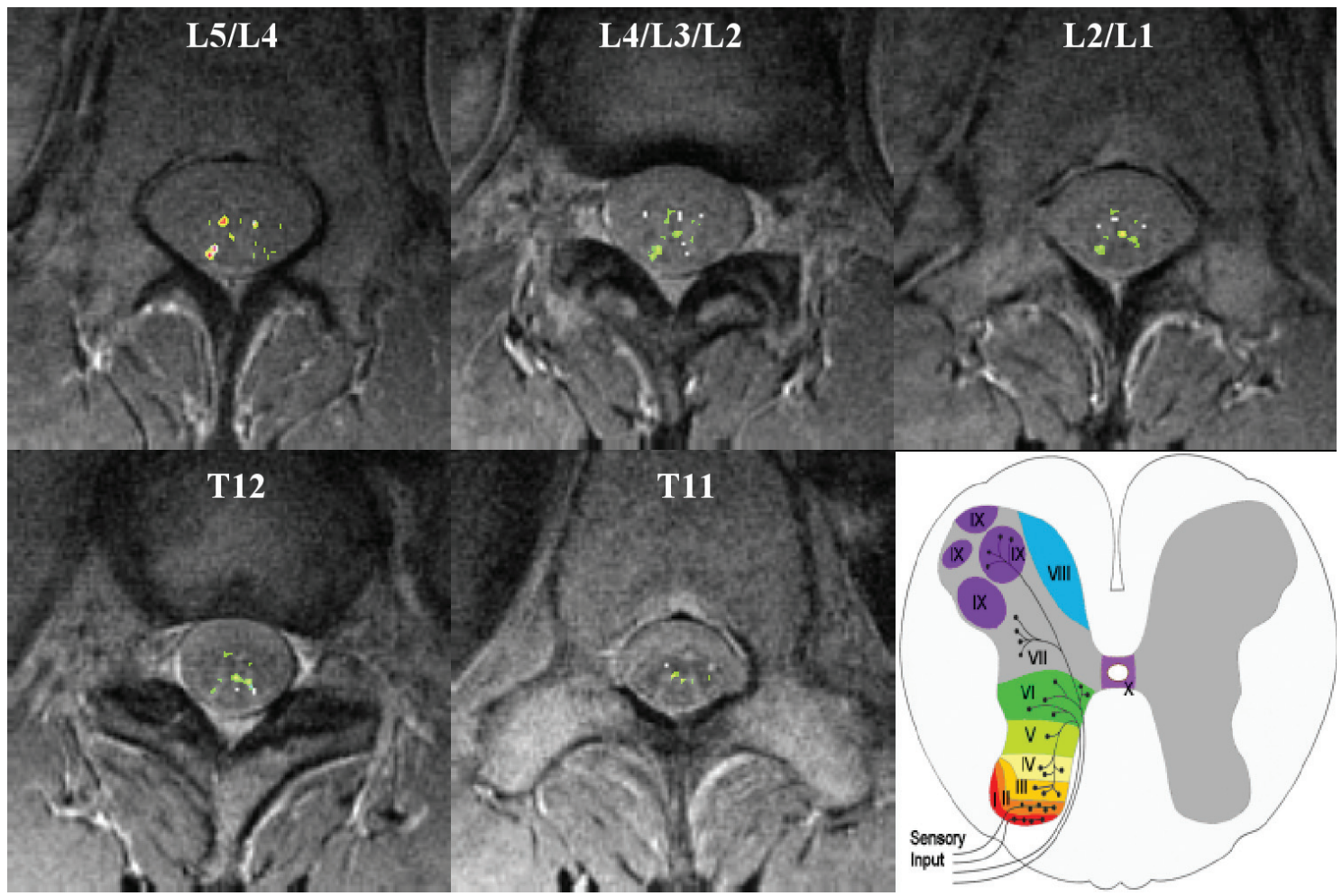

Figure 4. Combined data from the same subjects and same region of the spinal cord shown in figure 3 , demonstrating regions of activity arising only in the latter $24.75 \mathrm{sec}$ of the $33 \mathrm{sec}$ duration cold stimulation at $10^{\circ} \mathrm{C}$. The color scale used for the consistent areas of activity and the schematic inlay are the same as that used in figure 3 . Again, images are oriented with the right side of the body to the left, and the dorsal aspect of the spine toward the bottom of the images. 


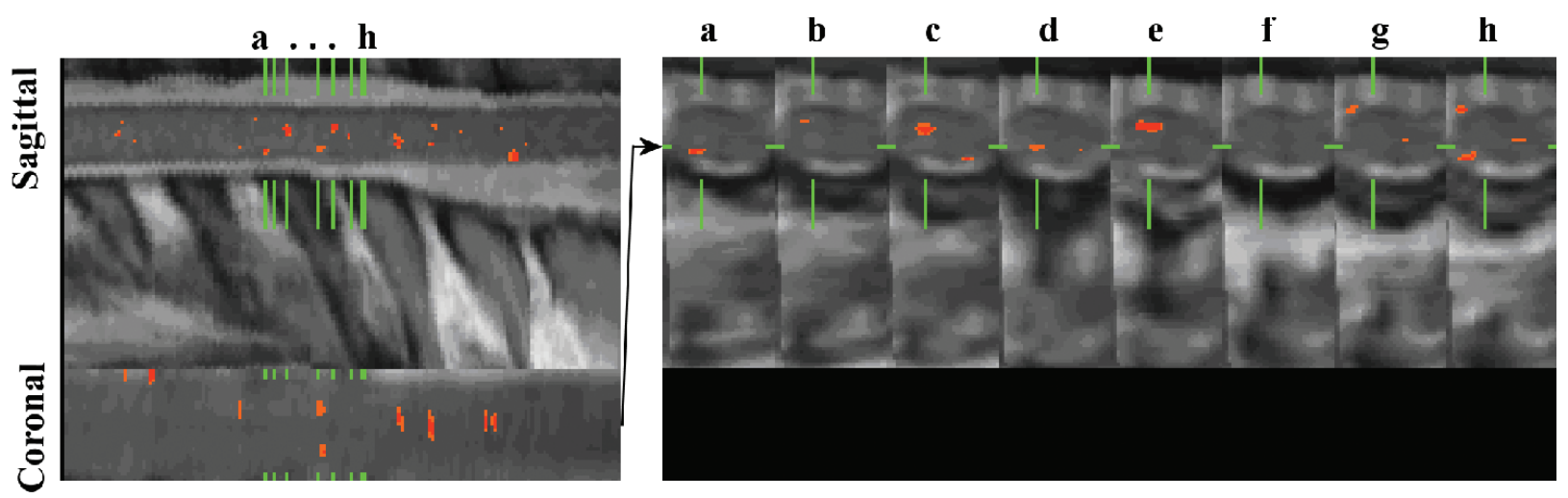

Figure 5. Example of one set of normalized results obtained from a single experiment with data obtained in thin contiguous sagittal slices. The figure shows a number of axial slices (right side) and one selected sagittal and coronal slice (as indicated on the left). The positions of the axial slices are indicated in the sagittal and coronal views with green lines, and the positions of the sagittal and coronal slices are indicated in each axial slice. Axial slices are in radiological orientation with the right side of the bottom toward the left side of the frame and dorsal is toward the bottom. Sagittal and coronal slices are oriented with rostral toward the left. Dorsal is toward the bottom in the sagittal slice and the left side of the body is toward the bottom of the coronal slices. The axial slices are in order from rostral to caudal, from left to right, and demonstrate the alternated pattern of dorsal (sensory) and ventral (motor) activity within a single spinal cord segment.

al. ${ }^{40}$ to determine the effects of retrospective cardiac gating; the results demonstrated that the majority of noise in spinal fMRI appears to be cardiac in origin. Given that all the causes of spinal cord motion within the spinal canal (CSF and blood flow) are driven by the cardiac motion, this conclusion seems highly plausible and may lead to a substantial improvement in the reliability and sensitivity of spinal fMRI results. It remains to be determined whether synchronizing the image acquisition with the heartbeat is sufficient or if there is an optimal time window in the cardiac cycle for data acquisition. If the latter is the case, then it is possible that this window will depend on the rostral-caudal position within the cord as pressure waves within the CSF propagate along the spinal canal.

From the studies reported to date, it appears that the optimal spinal fMRI method will employ an appropriate cardiac-gated acquisition scheme and will have secondary improvement in quality from the use of flow compensation gradients and spatial saturation pulses to eliminate interference from motion artifacts from surrounding tissues and fluids.

\section{Evidence that Spinal fMRI Demonstrates Areas of Neuronal Activity}

Seven different groups reporting spinal fMRI studies on human subjects have demonstrated that areas of signal change correlated with the stimulation paradigm were detected in locations corresponding with the expected areas of activity based on the neuroanatomy. ${ }^{6,7,33,40-43}$ Studies with animals (rats) have also been reported and will be discussed separately below. Evidence that the observed signal changes are related to neuronal activity is provided by the correspondence between the patterns of activity and the neuroanatomy. Studies carried out in our lab have outlined the detailed distribution of activity in the gray matter demonstrating activity primarily near the end of the dorsal horn ipsilateral to the stimulus as well as in ipsilateral motor areas (figure 3). 33 The pattern demonstrated matches the neuroanatomy of the spinal cord very closely. In the same study, the data also demonstrated areas of activity which arose only after the $10^{\circ} \mathrm{C}$ thermal stimulus had been applied for several seconds and had become noxious; activity was observed at the tip of the dorsal horn (near laminae I and II), in localized motor areas in the ipsilateral ventral gray matter and around the central canal (figure 4). More recent studies with sagittal slices have also demonstrated details of the sub-segmental distribution of activity in the rostral-caudal direction (figure 5). A similar pattern of activity has been demonstrated in electrophysiological studies in rats; 44 the cross-sectional pattern of activity is consistent with that observed in previous studies, but the fine-scale rostral-caudal distribution has not been previously demonstrated in humans. Studies employing stimulation of various sensory dermatomes on the hand and forearm in human subjects have also demonstrated a close correspondence

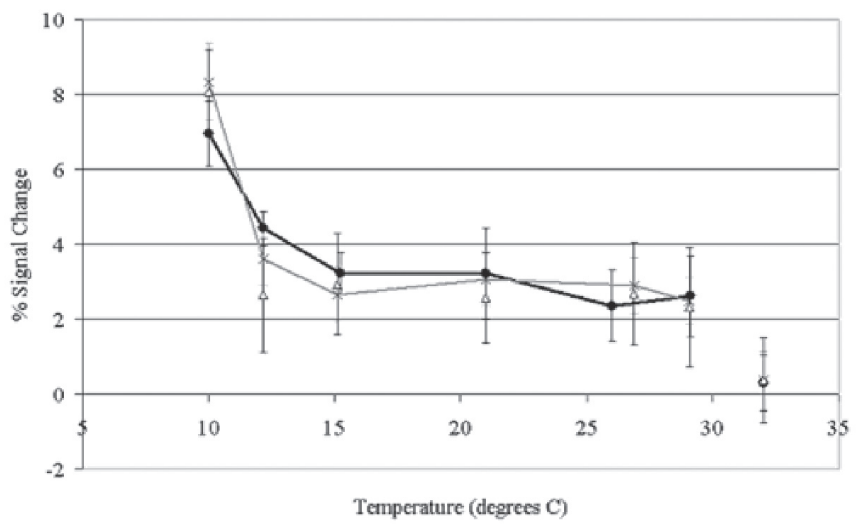

Figure 6. Spinal fMRI signal intensity response in the lumbar spinal cord to stimulation of the $L 4$ dermatome over a range of temperatures. Black circles indicate results from healthy volunteers, $\times$ symbols indicate results from subjects with complete spinal cord injuries, and triangles indicate results from subjects with incomplete injuries to the spinal cord. Signal intensity changes are relative to the baseline temperature at $32^{\circ} \mathrm{C}$. 

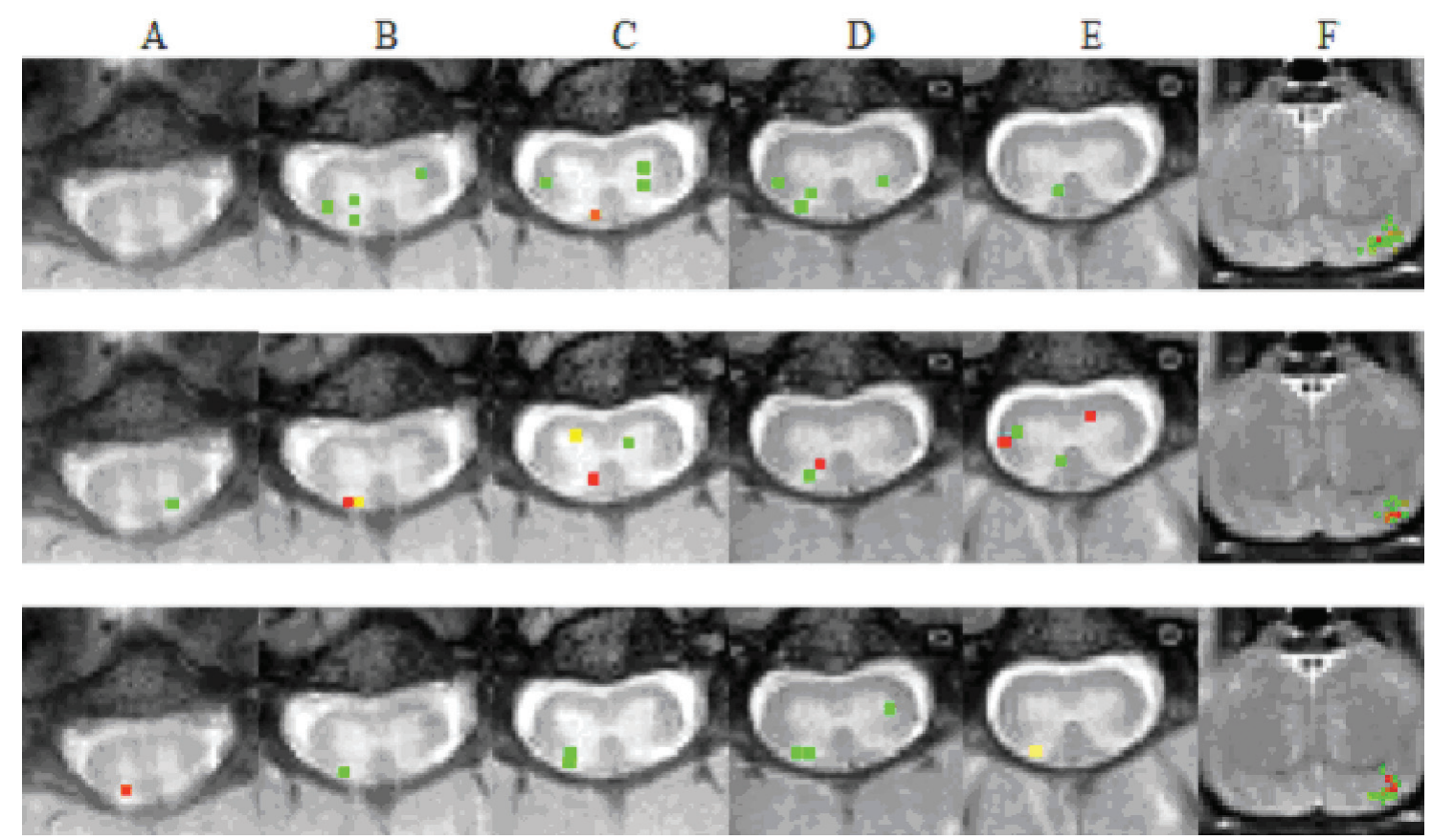

Figure 7. Areas of neuronal activity detected in the spinal cord (A-E) and brain (F) obtained simultaneously in the three functional $\mathrm{MRI}$ experiments in the same rat (rows 1 to 3, respectively). The spinal images are superimposed on anatomical images at five different levels of the cervical spinal cord: images corresponding to T2/T1, T1/C8, C7, C6 and C5 cervical levels from left to right (A-E) respectively with the ventral surface at the top, dorsal surface at the bottom. Left-right direction is according to radiology convention. The color of the activation corresponds to the level of the correlation to the paradigm: red corresponds to the highest, yellow to medium, and green the lowest correlation coefficients, respectively (all $P<0.001$ ). (Courtesy of K. Majcher et al. ${ }^{51}$ )

between the area of stimulation and the segmental level of activity observed in the spinal cord. ${ }^{39}$ Similarly, studies employing thermal stimulation of the leg have demonstrated corresponding activity in the lumbar spinal cord with differences demonstrated between the patterns of activity seen with thermal sensory stimulation and a motor task. ${ }^{33,45}$ Spinal fMRI studies of people with spinal cord injuries and with multiple-sclerosis have consistently demonstrated patterns of activity that are different than those seen in healthy subjects and that correspond with the injury or sensory deficits at the time of the study. ${ }^{38,46}$ All these examples support the conclusion that the areas of activity observed with spinal fMRI are indeed related to the neuronal function in the spinal cord.

However, more direct evidence has been obtained in studies comparing the magnitude of signal changes and stimuli of varying intensities. Madi et al. ${ }^{6}$ reported that signal intensity changes in the spinal cord depend on the force applied by muscles during an isometric motor task and concluded that the signal intensity changes observed were directly proportional to the force applied by the muscle group. Similarly, we compared the magnitude of signal intensity changes in the spinal cord with the temperature of a thermal stimulus applied to the leg over the range of $32^{\circ} \mathrm{C}$ to $10^{\circ} \mathrm{C}$. The signal intensity changes observed in active regions of the spinal cord averaged $2.6 \% \pm 1.1 \%$ (mean \pm S.D.) at around $29^{\circ} \mathrm{C}$, increasing marginally to $3.2 \% \pm 0.5 \%$ at $15^{\circ} \mathrm{C}$ and then increasing dramatically at lower temperatures to $7.0 \% \pm 0.9 \%$ at $10^{\circ} \mathrm{C}$ (figure 6). ${ }^{33}$ The response pattern was essentially identical in active regions of the spinal cord detected in spinal cord injured subjects. ${ }^{47}$ This bi-phasic response pattern corresponds well with the transition between cold thermal stimulation above $15^{\circ} \mathrm{C}$ to a noxious cold stimulation below $15^{\circ} \mathrm{C}$. During all these studies, the subjects participated only passively and serial time-course images were obtained of the lumbar spinal cord while the temperature of the thermode against the skin below the knee was varied in time. The only link between the temperature of the thermode and the images obtained of the spinal cord is via the input to the spinal cord from the receptors in the skin. These results, and those reported by Madi et al., 6 therefore provide direct evidence that the signal changes detected in spinal fMRI correspond with neuronal activity in the spinal cord.

\section{Animal Studies}

A relatively small number of animal studies have been reported in the literature but provide the most direct evidence to date that spinal fMRI results demonstrate areas of neuronal activity in the spinal cord. The first published animal spinal fMRI study 31 employed several key features of current methods: 1) data were acquired at $4.7 \mathrm{~T}$ with a fast spin-echo imaging method but 2 ) a relatively long effective echo time (100 msec) in order to detect the BOLD effect and 3) images were obtained from thin $(1 \mathrm{~mm})$ coronal slices with a high in-plane resolution $(0.12 \mathrm{~mm} \times 0.23 \mathrm{~mm})$. Malisza et al. ${ }^{48,49}$ have since demonstrated consistent results in rats at $9.4 \mathrm{~T}$ with a fast spin-echo, effective echo time of $85 \mathrm{msec}, 2 \mathrm{~mm}$ thick 
transverse slices and in-plane resolution of $0.16 \mathrm{~mm} \times 0.31$ $\mathrm{mm}$. All these studies used injection of a noxious substance (formalin or capsaicin) into the paw with fairly consistent results. Porszasz et al. ${ }^{31}$ demonstrated signal changes of $12.7 \%$ upon stimulation and Malisza et al. ${ }^{48,49}$ reported signal changes of $16 \%$ and $20 \%$. Lawrence et al. ${ }^{50}$ have also carried out a study to compare spinal fMRI results with direct histological assessment of areas of activity using c-fos staining. This study demonstrated good correspondence between areas of activity detected with spinal fMRI and those observed with c-fos labeling in the same animals with noxious electrical stimulation of the forepaw and hindpaw. Finally, a recent study by Majcher et al. ${ }^{51}$ has demonstrated simultaneous brain and spinal cord fMRI in rats during electrical stimulation of the forepaw (figure 7). Image data from both the brain and spinal cord were obtained in a single acquisition with a fast spin-echo at $9.4 \mathrm{~T}$ with an echo time of $44 \mathrm{msec}, 2 \mathrm{~mm}$ thick transverse slices and an in-plane resolution of $0.31 \mathrm{~mm} \times 0.31 \mathrm{~mm}$. In each study, the results demonstrated highly reproducible areas of activity in both the brain and cervical spinal cord in areas corresponding with the neuroanatomy. The magnitude of signal changes in both regions was approximately $3 \%$.

\section{Conclusions and Future Directions}

The current literature on spinal fMRI demonstrates both effective methods for data acquisition and that results obtained by these methods correspond with areas of neuronal activity in the spinal cord. One key step remains before spinal fMRI can be used as a tool for clinical assessment or for research: the reproducibility and reliability of the results this method provides must be determined. In all the spinal fMRI studies reported, there has been a degree of variability in the results, much like that seen in fMRI of the brain, but it is currently unknown how much variability is real and physiological, and how much is artifactual. In order to address this question a multi-site study is currently being conducted in healthy subjects to quantify the reproducibility of the results using the current spinal fMRI methods that we have established in our group. Preliminary results from this study demonstrate positive result reproducibility of at least $67 \%$ in active regions in spinal cord gray matter in single experiments, and that the occurrence of false positive results is lower than that of false negative results. The greatest source of error in spinal fMRI results can reasonably be expected to be cardiac in origin, as has been demonstrated by Brooks et al., ${ }^{40}$ however cardiac gating was not employed in the aforementioned reproducibility study. With the addition of cardiac gating and/or modelling of signal changes arising from cardiac motion to the established methods, the reproducibility of spinal fMRI results will be substantially improved. Although future improvements and advances are still to be expected, spinal fMRI methods, which are practical and effective for clinical assessment and spinal cord research, appear to be within reach.

\section{Acknowledgments}

I am grateful for the very helpful input to the preparation of this manuscript from Dr. Boguslaw Tomanek, Kasia Majcher, Dr. Spyros Kollias, Jennifer Kornelsen, Amanda Bergman, and last but not least, Jane Lawrence.

\section{References}

1. Ogawa S, Lee TM, Kay AR, Tank DW. Brain magnetic resonance imaging with contrast dependent on blood oxygenation. Proc Natl Acad Sci U S A 1990;87:9868-9872.

2. Ogawa S, Lee TM. Magnetic resonance imaging of blood vessels at high fields: in vivo and in vitro measurements and image simulation. Magn Reson Med 1990;16:9-18.

3. Ogawa S, Lee TM, Nayak AS, Glynn P. Oxygenation-sensitive contrast in magnetic resonance image of rodent brain at high magnetic fields. Magn Reson Med 1990;14:68-78.

4. Yoshizawa T, Nose T, Moore GJ, Sillerud LO. Functional magnetic resonance imaging of motor activation in the human cervical spinal cord. Neuroimage 1996;4:174-182.

5. Stroman PW, Nance PW, Ryner LN. BOLD MRI of the human cervical spinal cord at 3 tesla. Magn Reson Med 1999;42:571-576.

6. Madi S, Flanders AE, Vinitski S, Herbison GJ, Nissanov J. Functional MR imaging of the human cervical spinal cord. AJNR Am J Neuroradiol 2001;22:1768-1774.

7. Backes WH, Mess WH, Wilmink JT. Functional MR imaging of the cervical spinal cord by use of median nerve stimulation and fist clenching. AJNR Am J Neuroradiol 2001;22:1854-1859.

8. Stroman PW, Krause V, Malisza KL, Frankenstein UN, Tomanek B. Characterization of contrast changes in functional MRI of the human spinal cord at 1.5 T. Magn Reson Imaging 2001;19:833-838.

9. Bandettini PA, Wong EC, Jesmanowicz A, Hinks RS, Hyde JS. Spin-echo and gradient-echo EPI of human brain activation using BOLD contrast: a comparative study at $1.5 \mathrm{~T}$. NMR Biomed 1994;7:12-20.

10. Stroman PW, Krause V, Malisza KL, Frankenstein UN, Tomanek B. Extravascular proton-density changes as a non-BOLD component of contrast in fMRI of the human spinal cord. Magn Reson Med 2002;48:122-127.

11. Ugurbil K, Ogawa S, Kim S-G, Hu X, Chen W, Zhu X-H. Imaging brain function using nuclear spins. In: Maraviglia B, ed. Magnetic resonance and brain function: approaches from physics. Proceedings of the International School of Physics "Enrico Fermi". Societa Italiana di Fisica; Ohmsha, Amsterdam: IOS Press;1999. 261-301.

12. Stroman PW, Tomanek B, Krause V, Frankenstein UN, Malisza KL. Functional magnetic resonance imaging of the human brain based on signal enhancement by extravascular protons (SEEP fMRI). Magn Reson Med 2003;49:433-439.

13. Stroman PW, Kornelsen J, Lawrence J, Malisza KL. Functional magnetic resonance imaging based on SEEP contrast: Reproducibility, hemodynamic response function, and anatomical specificity. In: Proceedings of the International Society for Magnetic Resonance in Medicine 12th Annual Meeting, Kyoto, Japan, May 15-21, 2004: poster 2543.

14. Stroman PW, Malisza KL, Onu M. Functional magnetic resonance imaging at 0.2 Tesla. Neuroimage 2003;20:1210-1214.

15. Gati JS, Menon RS, Ugurbil K, Rutt BK. Experimental determination of the BOLD field strength dependence in vessels and tissue. Magn Reson Med 1997;38:296-302.

16. Kim SG. Quantification of relative cerebral blood flow change by flow-sensitive alternating inversion recovery (FAIR) technique: application to functional mapping. Magn Reson Med 1995;34:293-301. 
17. Fujita H, Meyer E, Reutens DC, Kuwabara H, Evans AC, Gjedde A. Cerebral [15O] water clearance in humans determined by positron emission tomography: II. Vascular responses to vibrotactile stimulation. J Cereb Blood Flow Metab 1997; 17:73-79.

18. Ohta S, Meyer E, Fujita, Reutens DC, Evans A, Gjedde A Cerebral $[15 \mathrm{O}]$ water clearance in humans determined by PET: I. Theory and normal values. J Cereb Blood Flow Metab 1996;16:765-780.

19. Bouzier-Sore AK, Merle M, Magistretti PJ, Pellerin L. Feeding active neurons: (re)emergence of a nursing role for astrocytes. J Physiol Paris 2002;96:273-282.

20. Nicholls JG, Martin AR, Wallace BG. Properties and functions of neuroglial cells. In: Nicholls JG, ed. From neuron to brain 3rd ed. Sunderland, Massachusetts: Sinauer Associates, Inc.;1992. 146-183.

21. Pellerin L, Magistretti PJ. Food for thought: challenging the dogmas. J Cereb Blood Flow Metab 2003;23:1282-1286.

22. Nedergaard M, Takano T, Hansen AJ. Beyond the role of glutamate as a neurotransmitter. Nat Rev Neurosci 2002;3:748-755

23. Piet R, Vargova L, Sykova E, Poulain DA, Oliet SH. Physiological contribution of the astrocytic environment of neurons to intersynaptic crosstalk. Proc Natl Acad Sci U S A 2004;101:2151-2155.

24. Amiry-Moghaddam M, Ottersen OP. The molecular basis of water transport in the brain. Nat Rev Neurosci 2003;4:991-1001.

25. Sykova E, Vargova L, Kubinova S, Jendelova P, Chvatal A. The relationship between changes in intrinsic optical signals and cell swelling in rat spinal cord slices. Neuroimage 2003;18:214-230.

26. Sykova E. Diffusion properties of the brain in health and disease. Neurochem Int 2004;45:453-466.

27. Brodal P. The Central Nervous System: Structure and Function. 2nd ed. New York, NY: Oxford University Press; 1998. 20.

28. Asai T, Kusudo K, Ikeda H, Murase K. Intrinsic optical signals in the dorsal horn of rat spinal cord slices elicited by brief repetitive stimulation. Eur J Neurosci 2002;15:1737-1746.

29. Anderson TR, Andrew RD. Spreading depression: imaging and blockade in the rat neocortical brain slice. J Neurophysiol 2002;88:2713-2725

30. Andrew RD, MacVicar BA. Imaging cell volume changes and neuronal excitation in the hippocampal slice. Neuroscience 1994;62:371-383.

31. Porszasz R, Beckmann N, Bruttel K, Urban L, Rudin M. Signal changes in the spinal cord of the rat after injection of formalin into the hindpaw: characterization using functional magnetic resonance imaging. Proc Natl Acad Sci U S A 1997;94:5034-5039.

32. Stroman PW, Kornelsen J, Lawrence J. An improved method for spinal functional MRI with large volume coverage of the spinal cord. J Magn Reson Imaging 2005;21:520-526.

33. Stroman PW, Tomanek B, Krause V, Frankenstein UN, Malisza KL. Mapping of neuronal function in the healthy and injured human spinal cord with spinal fMRI. Neuroimage 2002; 17:1854-1860.

34. Stroman PW, Ryner LN. Functional MRI of motor and sensory activation in the human spinal cord. Magn Reson Imaging 2001;19:27-32.

35. Matsuzaki $\mathrm{H}$, Wakabayashi $\mathrm{K}$, Ishihara $\mathrm{K}$, Ishikawa $\mathrm{H}$, Kawabata H, Onomura T. The origin and significance of spinal cord pulsation. Spinal Cord 1996;34:422-426.

36. Schumacher R, Richter D. One-dimensional Fourier transformation of M-mode sonograms for frequency analysis of moving structures with application to spinal cord motion. Pediatr Radiol 2004;34:793-797.
37. Mikulis DJ, Wood ML, Zerdoner OA, Poncelet BP. Oscillatory motion of the normal cervical spinal cord. Radiology 1994;192:117-121.

38. Stroman PW, Krause V, Malisza KL, Kornelsen J, Bergman A, Lawrence J, Tomanek B. Spinal fMRI of spinal cord injury in human subjects. In: Proceedings of the International Society for Magnetic Resonance in Medicine 11th Annual Meeting, Toronto, Canada, July 10-16, 2003: poster 13.

39. Stroman PW, Krause V, Malisza KL, Frankenstein UN, Tomanek B. Functional magnetic resonance imaging of the human cervical spinal cord with stimulation of different sensory dermatomes. Magn Reson Imaging 2002;20:1-6.

40. Brooks J, Robson M, Schweinhardt P, Wise R, Tracey I. Functional magnetic resonance imaging (fMRI) of the spinal cord: a methodological study. In: American Pain Society 23rd Annual Meeting, Vancouver, BC Canada, May 6-9, 2004: poster 667. Available at: http://www.ampainsoc.org/abstract/2004/data/667/index.html. Last accessed July 29, 2005.

41. Kollias SS, Kwiecinski S, Summers P. Functional MR Imaging of the Human Cervical Spinal Cord. In: American Society of Neuroradiology, Proceedings of the 42nd Annual Meeting, Seattle, June 7-11, 2004: poster 227. Available at: http://www.abstractsonline.com/viewer/viewAbstractPrintFrie ndly.asp?CKey $=\{16 \mathrm{E} 2 \mathrm{D} 87 \mathrm{~F}-\mathrm{B} 378-4556-9699$ -

$774561 \mathrm{DB} 7707\} \&$ SKey $=\{053974 \mathrm{D} 8-783 \mathrm{~F}-4 \mathrm{BF} 1-9973$ $3075 \mathrm{~A} 326 \mathrm{BFCF}\} \& \mathrm{MKey}=\{\mathrm{ED} 255145-0562-4 \mathrm{CAC}-\mathrm{AC} 4 \mathrm{~F}-$ $\mathrm{F} 1 \mathrm{E} 94 \mathrm{E} 8 \mathrm{D} 05 \mathrm{~F} 6\} \& \mathrm{AKey}=\{1 \mathrm{CC} 9 \mathrm{ED} 8 \mathrm{~F}-\mathrm{E} 877-432 \mathrm{D}-98 \mathrm{~B} 3-$ 8BB6898BEE36 \}. Last accessed July 29, 2005.

42. Mackey S, Ludlow D, Knierim J, Hanelin J, Glover G. FMRI activation in the human cervical spinal cord to noxious thermal stimulation. In: American Pain Society 22nd Annual Meeting, Chicago, March 20-23, 2003: poster 750. Available at: http://www.ampainsoc.org/abstract/2003/data/750/index.html. Last accessed July 29, 2005.

43. Wong KK, Ng MC, Hu Y, Luk DK, Ma QY, Yang ES. Functional MRI of the spinal cord at low field. In: Proceedings of the International Society for Magnetic Resonance in Medicine 12th Scientific Meeting \& Exhibition, Kyoto, Japan, May 15-21, 2004: poster 1534.

44. Takahashi Y, Chiba T, Kurokawa M, Aoki Y. Dermatomes and the central organization of dermatomes and body surface regions in the spinal cord dorsal horn in rats. J Comp Neurol 2003;462:29-41.

45. Kornelsen J, Stroman PW. fMRI of the lumbar spinal cord during a lower limb motor task. Magn Reson Med 2004;52:411-414.

46. Bergman A, Leblanc C, Stroman PW. Spinal fMRI of multiple sclerosis in human subjects. In: Proceedings of the International Society for Magnetic Resonance in Medicine 12th Scientific Meeting \& Exhibition, Kyoto, Japan, May 15-21, 2004: poster 1126.

47. Stroman PW, Kornelsen J, Bergman A, Krause V, Ethans K, Malisza KL, Tomanek B. Noninvasive assessment of the injured human spinal cord by means of functional magnetic resonance imaging. Spinal Cord 2004;42:59-66.

48. Malisza KL, Stroman PW. Functional imaging of the rat cervical spinal cord. J Magn Reson Imaging 2002;16:553-558.

49. Malisza KL, Stroman PW, Turner A, Gregorash L, Foniok T, Wright A. Functional MRI of the rat lumbar spinal cord involving painful stimulation and the effect of peripheral joint mobilization. J Magn Reson Imaging 2003;18:152-159.

50. Lawrence J, Stroman PW, Bascaramurty S, Jordan LM, Malisza KL. Correlation of functional activation in the rat spinal cord with neuronal activation detected by immunohistochemistry. Neuroimage 2004;22:1802-1807. 
51. Majcher K, Tomanek B, Jasinski A, Foniok T, Stroman PW, Kirk D, Tuor UI, Hess G. Simultaneous functional magnetic resonance imaging in the rat spinal cord and brain. In: Proceedings of the International Society for Magnetic Resonance in Medicine 13th Annual Meeting, Miami, USA, May 7-13, 2005: poster 1982.

\section{Author Affiliations}

Patrick W. Stroman, PhD, Department of Diagnostic Radiology, c/o Center for Neuroscience Studies, 231 Botterell Hall,

Queen's University, Kingston, Ontario, Canada K7L 2 V7. 